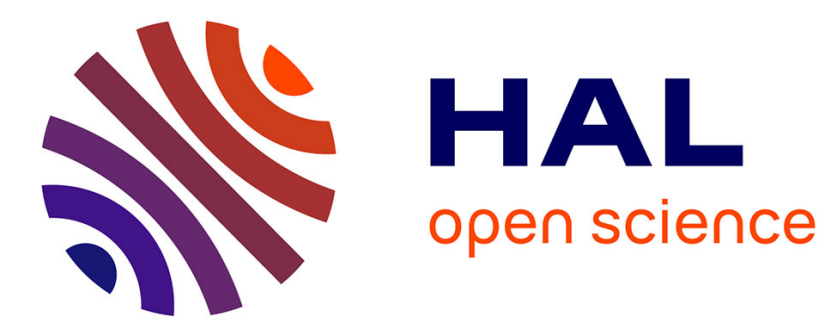

\title{
MPTCP Solution for Seamless Local SIPTO Mobility
}

Souheir Eido, Pratibha Mitharwal, Annie Gravey, Christophe Lohr

\section{To cite this version:}

Souheir Eido, Pratibha Mitharwal, Annie Gravey, Christophe Lohr. MPTCP Solution for Seamless Local SIPTO Mobility. HPSR 2015: 16th International Conference on High Performance Switching and Routing, Jul 2015, Budapest, Hungary. pp.1 - 6, 10.1109/HPSR.2015.7483099 . hal-01337291

\section{HAL Id: hal-01337291 \\ https://hal.science/hal-01337291}

Submitted on 4 Sep 2018

HAL is a multi-disciplinary open access archive for the deposit and dissemination of scientific research documents, whether they are published or not. The documents may come from teaching and research institutions in France or abroad, or from public or private research centers.
L'archive ouverte pluridisciplinaire HAL, est destinée au dépôt et à la diffusion de documents scientifiques de niveau recherche, publiés ou non, émanant des établissements d'enseignement et de recherche français ou étrangers, des laboratoires publics ou privés. 


\title{
MPTCP Solution for Seamless Local SIPTO Mobility
}

\author{
Souheir EIDO, Pratibha MITHARWAL, Annie GRAVEY, Christophe LOHR
}

\begin{abstract}
In this paper, we propose a method to support seamless mobility for sessions carried by SIPTO connections. Session continuity has been considered an issue to be solved within 3GPP, as none of the solutions proposed for Mobile IP directly apply in the context of LTE. As in some cases, typically when SIPTO relies on using Local Gateways (LGW), it is necessary to change the IP address allocated to a User Equipment (UE), an active session may be interrupted. We propose an MPTCP based solution within the LTE architecture to maintain a single session, initially carried over a given SIPTO connection, and then carried over another SIPTO connection initiated due to the mobility of user equipment (UE). We identify how MPTCP operates over the LTE architecture, and estimate whether the corresponding delay is compatible with session continuity.
\end{abstract}

Keywords- SIPTO, MPTCP, Mobility, Session Continuity, Mobile Data Offloading

\section{INTRODUCTION}

$\mathrm{I}^{\mathrm{I}}$ n the past twenty years, mobile networks have gone through several generations seeking, from one generation to another, to provide better throughput and higher capacity. The principal goal is to improve the user experience with the least possible investment on the infrastructure. Today, Long Term Evolution/System Architecture Evolution (LTE/SAE) network represents the last known mobile generation, i.e. " 4 th Generation". The LTE/SAE network uses an End-to-End (E2E) tunnel between the User Equipment (UE) and the Packet Data Network Gateway (PGW) [1]. The latter then allows the users to access the IP backbone and the Internet.

An E2E tunnel is divided in three different segments:

- Radio Tunnel: used to exchange data between the UE and either the radio base station "evolved Node B (eNB) for macrocells" or "Home eNodeB (HeNB) for femtocells" using the LTE-Uu interface.

The research leading to these results has received funding from the European Community Seventh Framework Program FP7/2013- 2015 under grant agreement no. $317762 \mathrm{COMBO}$ project.

Souheir EIDO, Pratibha MITHARWAL, Annie GRAVEY are with Institut Télécom, Télécom Bretagne and UMR CNRS 6074 IRISA. Technopôle Brest Iroise CS 83818 ; 29238 Brest Cedex 3 France

(email:firstname.lastname@telecom-bretagne.eu).

Christophe LOHR is with Institut Télécom, Télécom Bretagne and UMR CNRS 6285 Lab-STICC. Technopôle Brest Iroise CS 83818; 29238 Brest

Cedex 3 France (email:christophe.lohr@telecom-bretagne.eu).
- Backhaul Tunnel: used to exchange data between the (H)eNB and the Serving Gateway (SGW) using the S1-U interface.

- Core Tunnel: used to exchange data between the SGW and the PGW using S5/S8 interface.

The use of an E2E tunnel allows a secure connection and a seamless UE mobility with high QoS. However, even when a user requests a content that is geographically close, the requested traffic must first be sent to the PGW in the EPC network (Evolved Packet Core) and then be tunneled to be sent towards the UE. This sub-optimal routing leads to wasting network resources.

The Selected IP Traffic Offload (SIPTO) approach has been proposed by $3 \mathrm{GPP}$ in [2] in order to selectively breakout some of the mobile IP traffic closer to the UE, using femtocells or macrocells with PDN gateways that are either co-located with the radio base station or represented as standalone entities. SIPTO allows the selected IP traffic to be directly routed within the fixed access/metro networks while non-offloaded traffic is routed towards the EPC network. According to [3], the use of SIPTO can potentially save more than $30 \%$ of the bandwidth used in backbone as well as $15 \%$ of the bandwidth used in the metro/core segment of the network.

Mobile networks are intended to support seamless mobility. However, 3GPP has identified in [2] and [4] a few mobility use cases in the framework of SIPTO, where service continuity is not supported because the IP address of the UE has been changed due to its mobility.

In the present paper, we assess whether a solution proposed by IETF for managing multiple allocated IP addresses to a single host can be used to provide this seamless mobility. IETF has specified both Multi-Path Transmission Control Protocol (MPTCP) and Concurrent Multipath Transfer Stream Control Transmission Protocol (CMT-SCTP). MPTCP and CMT-SCTP can hopefully help solving session continuity issue for mobile users. As MPTCP is compatible with existing applications, the present paper focuses on its application to solve session continuity.

MPTCP enables any host to use multiple available network interfaces simultaneously for a single TCP session. Each interface carries a sub-flow of a single TCP connection presented to the application layer. The use of multiple available network interfaces can improve throughput by spreading data traffic over different network interfaces. This 
multi-homing property of MPTCP can be used during mobility of UEs. During mobility, an initial IP address can become unreachable as the UE moves out of network range whereas another IP address can be obtained through a new attachment. If MPTCP sub-flows are associated to each of these addresses, handover is facilitated and this can help in maintaining session continuity during UE relocation.

The present paper assesses whether an MPTCP-based solution can indeed support seamless mobility for users with activated SIPTO PDN-connections.

The rest of the paper is organized as follows. Section 2 presents the state of the art for SIPTO and MPCP; it also discusses SIPTO service continuity. Section 3 proposes an MPTCP-based SIPTO solution for session continuity. This proposal is evaluated in Section 4. Finally, Section 5 concludes the paper.

\section{STATE OF THE ART}

This section presents an overview of SIPTO for mobile data offloading. It then presents some mobility use cases where session continuity for users with multiple IP addresses can be an issue. Finally, the basics of MPTCP operation are outlined.

\section{A. SIPTO Architecture}

According to 3GPP in [1] and IETF in RFC 6459 [5], a UE can only be connected to one SGW at a time. At the establishment of SIPTO PDN connection, the Mobility Management Entity (MME) selects its "preferred" SGW.

SIPTO was first defined within the 3GPP SA2 group in order to breakout selected IP traffic (e.g., Internet) at or above the RAN [2]. In particular, SIPTO allows alleviating the loads on the mobile aggregation and core networks regardless of the available radio access network (i.e., Macrocells, Picocells or Femtocells). The basic idea is to select a SGW and a PGW that are topologically/geographically close to the UE. SIPTO above RAN relies on the same architecture model and concepts of LTE/SAE architecture described in clause 4.2 in [1]. Consequently, the selected SGW and PGW might either be combined together in a single gateway or separated from each other.

SIPTO has been extended by 3 GPP in [4] in order to support breaking out a selected IP traffic within the residential or enterprise IP network, or at local network (@LN). SIPTO (a) LN allow a UE to directly access the private IP network services using an eNB or even a HeNB with a co-located or a standalone Local Gateway (LGW) [6]. The LGW is also the gateway towards the external IP network. It supports especially PGW functions such as UE's IP address allocation and DHCPv4 functions. Moreover, LGW supports some of the SGW's functions such as downlink packet buffering as well as direct tunneling towards the $(\mathrm{H}) \mathrm{eNB}$, although it is not a full SGW since the UE is already linked to a standalone SGW for its non-SIPTO traffic.

Fig. 1 illustrates the different SIPTO use cases where UEs are having regular LTE/SAE traffic routed towards EPC while offloading part of its IP traffic to download from a Video-onDemand (VoD) server, which is located within the IP network.
Traffic for UE 1 traverses the SGW for both the regular traffic and the offloaded one. However, only regular traffic from UE 2 traverses the SGW. As the LGW includes functionalities of both PGW and SGW, UE 2 is virtually connected to two SGWs simultaneously: the standard SGW is used by the nonSIPTO traffic and the LGW is used by the traffic offloaded thanks to SIPTO.

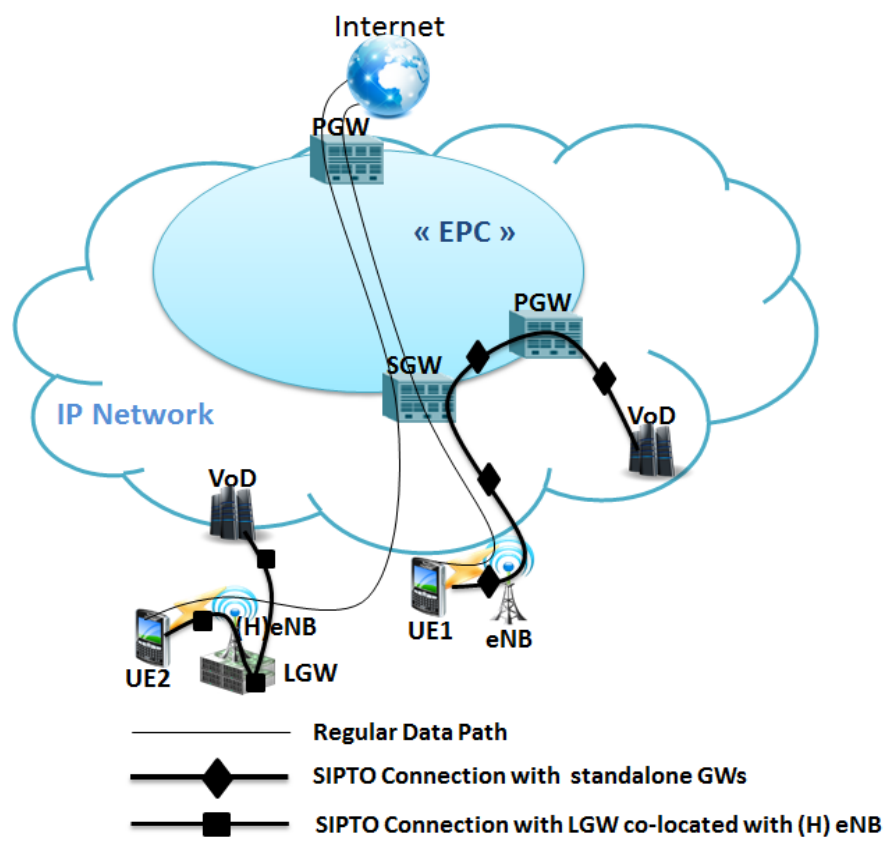

Fig. 1. Selected IP Traffic Offload Architecture

\section{B. Mapping LTE on network's topology}

In the currently deployed LTE architectures, network operators typically deploy a small number of SGWs and PGWs. This makes sense as long as the amount of data traffic carried over LTE is small.

However, as shown in [3], when the amount of data traffic (VoD and cloud-based traffic) grows to become dominant in both fixed and mobile networks, the network operator deploys a distributed content distribution architecture with data centers and $\mathrm{VoD}$ servers located closer to the users, typically in every large or medium town.

LGWs, co-located either with HGWs or eNBs are obvious breakout points that allow reaching those distributed data centers and VoD servers.

\section{Mobility Use Cases}

Depending on the type of GWs used to access the external IP network for SIPTO, 3GPP has distinguished two different mobility use cases:

1) SIPTO above RAN with standalone GWs (SGW/PGW): According to [4], session continuity for SIPTO above the RAN with standalone SGW and PGW is supported using the existing mobility procedures defined in $3 \mathrm{GPP}$ specifications for LTE/SAE architecture. This includes UE's mobility within Macro network, Femto network and between Macro and Femto cellular networks.

2) SIPTO with $L G W$ co-located with (H)eNB : As a result of UE mobility during an ongoing SIPTO session with either 
eNB or HeNB co-located with the LGW, a GW relocation procedure will be provided by the MME. Effectively, the relocation of SIPTO LGW will result in losing the IP address allocated by the initial LGW to the UE. Consequently, the MME must disconnect the impacted SIPTO PDN connection with re-connection cause required [1].

This procedure will likely be seamless for short-lived applications (e.g. SMS texting). However, the disconnection of current SIPTO PDN connection will affect mostly the services, which require IP address preservation such as e.g., Real Time Video Streaming and online gaming. As shown in Fig. 2, such services will be interrupted during all the UE's mobility procedure. After the reactivation of the new SIPTO PDN connection, the UE can re-connect to those services.
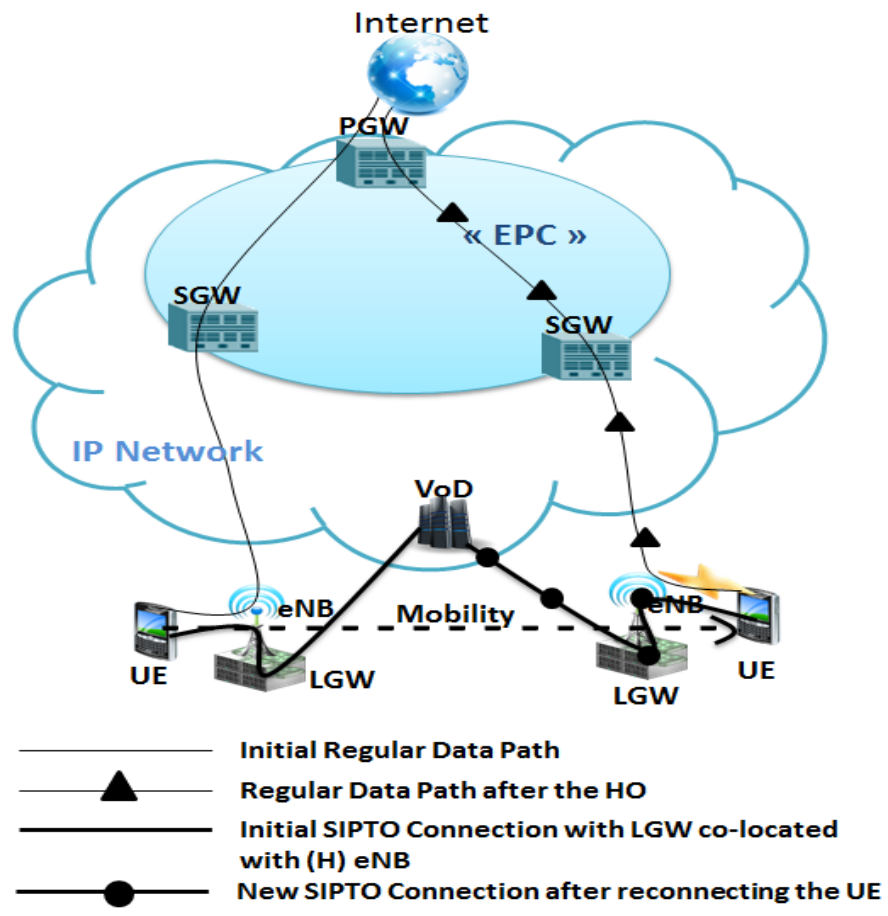

Fig. 2. 3GPP Mobility scenario for UE having a SIPTO Session with LGW co-located with $(\mathrm{H}) \mathrm{eNB}$

\section{MultiPath Transmission Control Protocol (MPTCP)}

MPTCP is an extension of TCP standardised in RFC 6824 [7]. It was originally proposed to provide support for multihomed hosts. It enables a mobile host to use multiple available interface connections simultaneously and thus allows multipath streaming [8]. Its target benefit is load balancing the traffic over different interfaces of a mobile host, which potentially results in improved throughput. MPTCP is backward compatible with TCP and uses the standard socket API used by most Internet applications, which makes it compatible with existing application and network.

MPTCP connection establishment starts as a standard TCP connection with SYN segment included with MPTCP option (MP-CAPABLE) to verify whether the receiving host supports MPTCP or not. If the receiving host or remote host supports
MPTCP, it will add the option in SYN-ACK reply. The two hosts also include cryptographic tokens to these packets to uniquely identify this connection. If there are more than one network interfaces available at the start of the connection the additional sub-flows can be added to this MPTCP connection with the final ACK. Each sub-flow behaves as a separate regular TCP connection inside the network. Sub-flows can be added and removed at any point of time, in any MPTCP ongoing communication, with the help of ADD_ADDR option and REMOVE_ADDR option for any interface. These options can be helpful during mobility of a mobile host when it moves from one network to another network i.e., it receives or configures a new IP address through new network attachment. MPTCP uses "make before break" method for seamless mobility, which maintains the ongoing communication using smooth handover.

MPTCP provides different handover modes, namely full handover mode, backup mode and single-path mode [9]. In full handover mode, all the sub-flows are used simultaneously between two communicating hosts. Whereas, in backup mode MPTCP opens sub-flows over all the existing interfaces but uses only a subset of sub flows for transmission of data packets. MPTCP uses MP-PRIO option to specify any subflow as backup mode. The sub-flow, which is defined in MPPRIO option, will be used only when the other addresses are not working. In the single-path mode only one TCP sub-flow is used at any time. If the interface goes down then another sub-flow can be created and used for packet forwarding.

In Fig. 3, the mobile Host $(\mathrm{MH})$ represents user equipment (UE) and Remote Host (RH) represents any peer node (e.g., video server). Here we assume that mobile host and remote host both have two IP addresses so in this case they can create 4 sub-flows through a full mesh architecture.

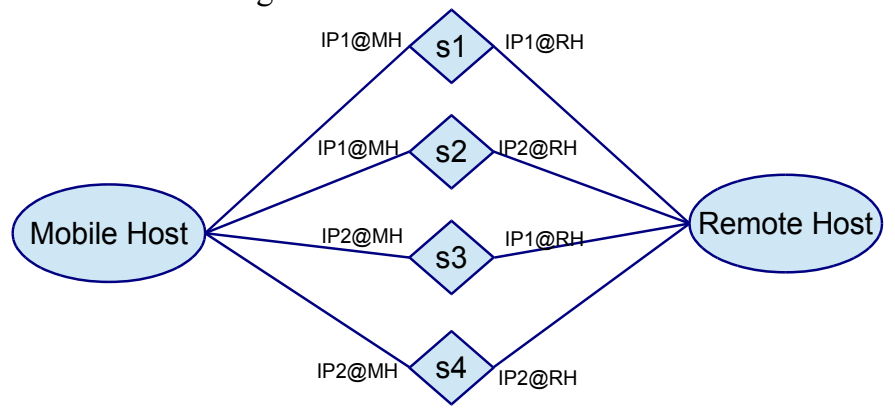

Fig. 3. Full Mesh Architecture for MPTCP communication

\section{E. Conclusion of State of Art}

As shown in Section $\mathrm{C}$, session continuity is not maintained during mobility of UEs with SIPTO@LN with a LGW colocated with the (H)eNB. Most of the current studies focusing on offloading mobile data traffic with seamless mobility are considering "IP address preservation" as a key issue for SIPTO approach. 


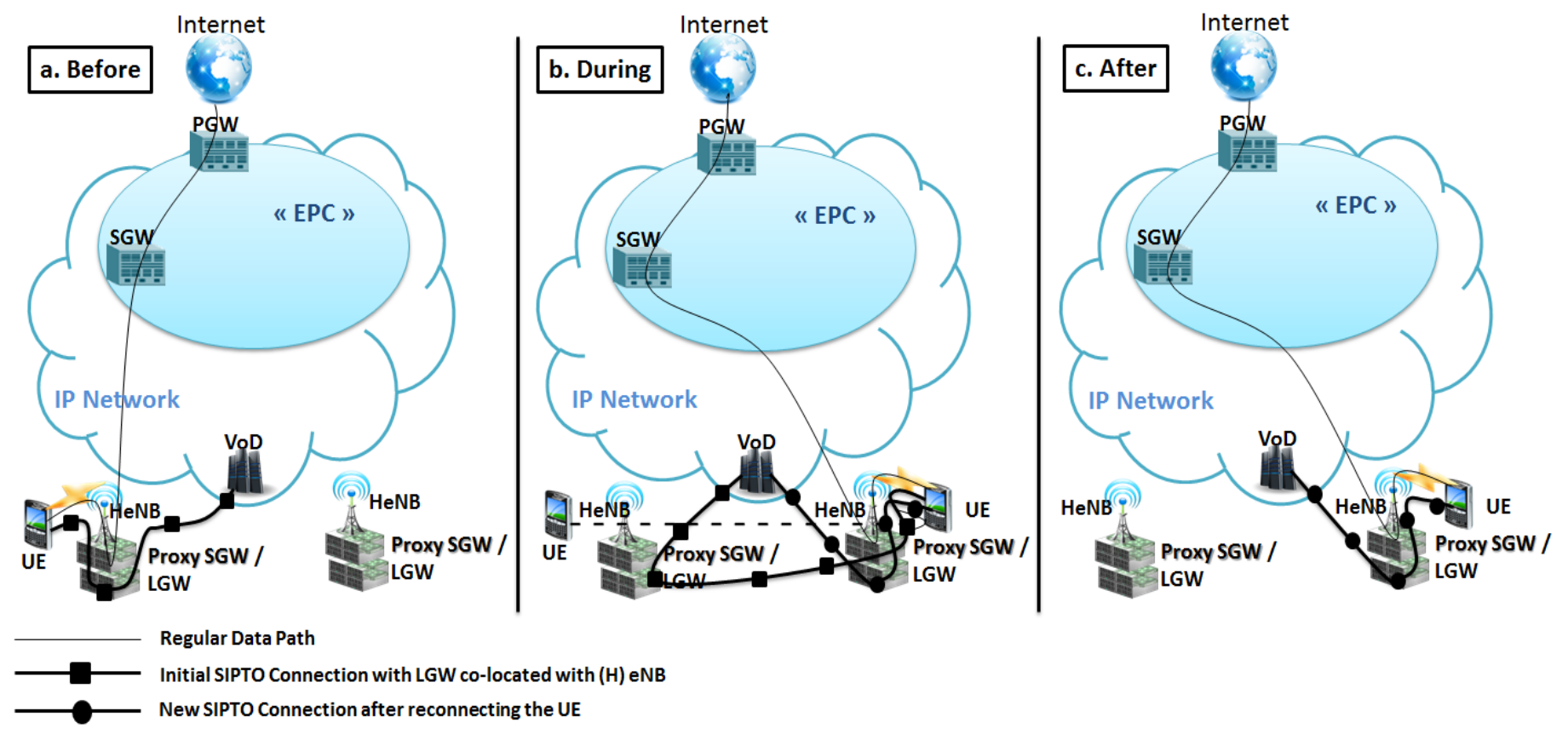

Fig. 4. Proposed Mobility Scenario for UE having Regular session and SIPTO session with Proxy SGW function included in LGW.

Existing solutions that have been considered have focused on offloading the short-lived IP traffic while routing the real time streaming traffic towards the EPC network e.g., C-SIPTO [10]. IETF proposes new techniques to avoid centralized mobility e.g., DMM [11]. This paper differs from the existing methods by introducing an MPTCP-based solution allowing a seamless mobility for SIPTO users. Our MPTCP-based solution aims at synchronizing the UE's ongoing session to be routed using several paths simultaneously while guaranteeing a backup path in case that one of the links breaks down. Moreover, to our best knowledge, none of the proposed solutions have yet considered using LGW with co-located (H)eNB as a solution to offload real time streaming video, which is proposed here.

\section{PROPOSED MPTCP SOLUTION}

In the standard 3GPP, the relocation of SIPTO LGW will result in losing the IP address allocated by the initial LGW to the UE, which thus loses its previous connection due to mobility. The UE then re-establishes a new session, during which it gets a new IP address to re-access the external IP network (e.g., VoD server). Since the VoD server is not aware that the previous IP address and the new IP address reach the same user, the traffic is interrupted between the deactivation and reactivation procedures.

When the UE establishes a MPTCP connection with MPTCP capable VoD server, the VoD server will be able to synchronize the user's traffic using different IP addresses distributed on MPTCP sub-flows. To achieve this, a handover procedure will be performed on the initial SIPTO connection while the new SIPTO connection is being established. After handover, the original SIPTO connection is deleted and all its allocated resources are released.

All MPTCP signaling is performed over the LTE regular
Data Path, which is always available, even during handover, thanks to existing LTE mobility procedures.

\section{A. Mobility MPTCP-based SIPTO with co-located GWS}

The LGW is considered by the 3 GPP mobile architecture as a PGW with some additional functionality, and not as a colocated SGW and PGW. We now introduce the notion of "Proxy-SGW". A Proxy-SGW represents a GW that is seen as:

- a regular SGW by both the $(\mathrm{H}) \mathrm{eNB}$ and the LGW

- a (H)eNB by the regular SGW.

Fig. 4 illustrates a use case of a UE that is connected to a HeNB with co-located LGW including Proxy-SGW functionality. Originally (Fig. 4-a), all upstream traffic received by the HeNB is intercepted by the Proxy-SGW which routes the regular traffic to the regular SGW and SIPTO traffic to the LGW.

Following a decision of GW relocation throughout UE's mobility procedure, the initial Proxy-SGW will also be relocated towards a new Proxy-SGW as shown in Fig. 4-b.

After the GW relocation decision, the handover procedure is carried out:

1. a HO procedure is performed to forward the regular traffic from the source HeNB (S-HeNB) to the target HeNB (THeNB).

2. an S1-based HO procedure is then performed to forward the SIPTO traffic from the source Proxy-SGW (S-ProxySGW) to the target Proxy-SGW (T-Proxy-SGW) and then from the T- Proxy-SGW to T-HeNB.

3. a new SIPTO PDN-Connection is established for the same ongoing session, in parallel to the first one. Thanks to the MPTCP connection "join option", the VoD server will add the new UE's IP address allocated to the UE by the target LGW (T-LGW), to its list of addresses for that UE. 
The VoD server will then know that all the IP addresses belong to the same user and synchronize the sub-flows, so that the UE will receive parts of the packets of the first session on both sub-flows: "the sub-flow of the previous SIPTO connection" and "the sub-flow of the new SIPTO connection".

Once the S1-based HO procedure is completed for the regular traffic and the new SIPTO PDN connection is finally established for the offloaded traffic, a deactivation procedure for the previous SIPTO PDN connection will be performed, all the IP addresses allocated by the source LGW will be deleted from the list of addresses stored at the VoD server and then all the allocated resources of the previous SIPTO PDN connection will be released. This is illustrated in Fig. 4-c.

\section{B. MPTCP Signaling over LTE Data Path}

The first step of MPTCP signaling starts with the MPTCP connection establishment. Whenever a UE requests the establishment of SIPTO PDN Connection, it receives the IP address of an MPTCP capable VoD server from the PGW and establishes an MPTCP connection. After the establishment of the MPTCP connection between UE and VoD server, we use the Backup Mode to specify the regular LTE path as "backup path" with MP-PRIO option. Once the SIPTO connection is established, the UE must ADD its SIPTO IP address to the ongoing MPTCP connection with VoD server. After the addition of SIPTO IP address it needs to start a regular subflow with this IP address for traffic offloading. MPTCP signaling is illustrated in Fig. 5.

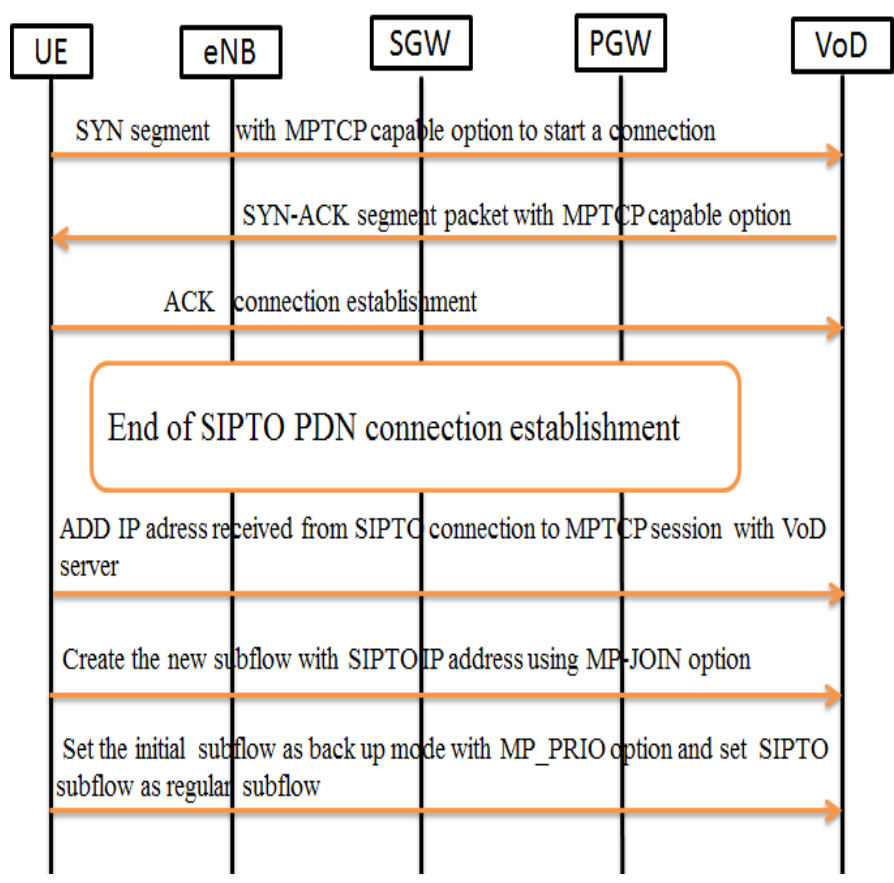

Fig. 5. MPTCP connection establishment between UE and VoD server.

During the UE mobility with LGW relocation, a new SIPTO PDN connection must be established with the target LGW. In order to maintain the ongoing session, the UE communicates its new IP address to the VoD server to add a new sub-flow to the ongoing MPTCP session before disconnecting the previous SIPTO connection. After the establishment of the new SIPTO MPTCP sub-flow, the UE may request the removal of its previous SIPTO IP address from the VoD server's IP list. This signaling procedure is also maintained using the regular LTE data path as illustrated in Fig. 6.

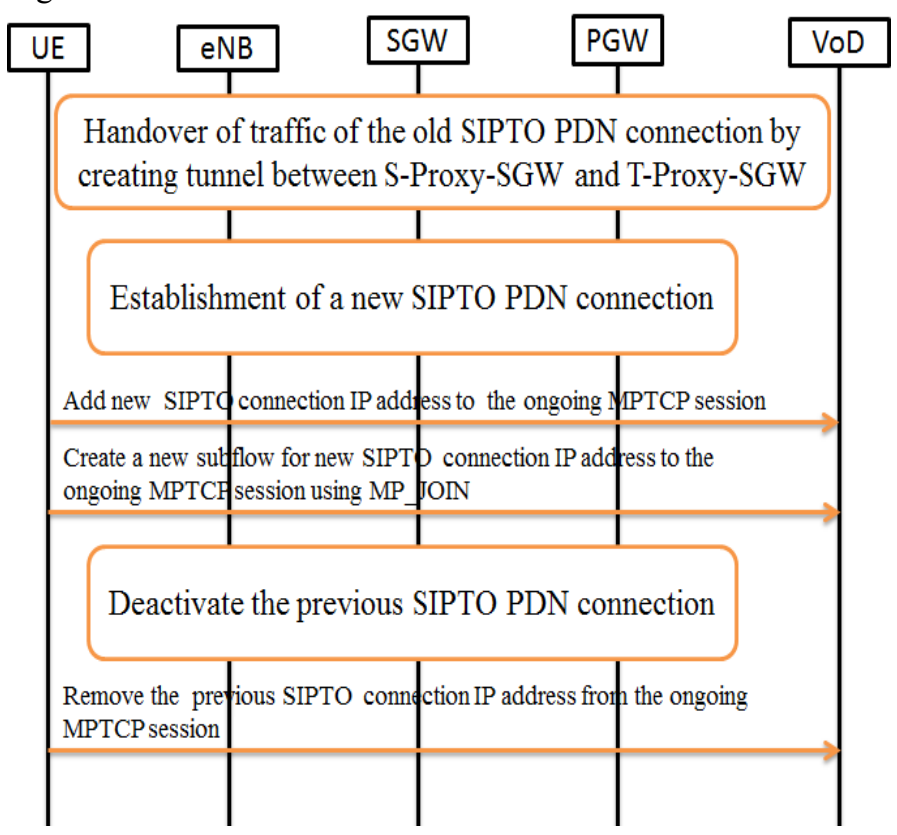

Fig. 6. MPTCP Add-address procedure during mobility of UE.

\section{EVALUATION OF THE SOLUTION}

In this section, the performance of our proposed MPTCPbased SIPTO scenario is evaluated in terms of interruption time duration with respect to service continuity. The evaluation is focused on finding out whether the proposed scenario can reduce the interruption time of SIPTO's traffic during the mobility of UEs between two cells with GW relocation decision triggered by the MME.

Let $\mathrm{PD}$ and LD respectively represent the processing delay of the nodes and the link transmission time between different nodes. Let also SDD, SED and TED respectively represent the SIPTO connection deactivation procedure delay, SIPTO connection establishment procedure delay and indirect tunnel establishment delay between S-Proxy-SGW and T-ProxySGW in proposed scenario for the S1-based HO.

The interruption time of SIPTO@LN traffic for standard mobility use case is given by:

$$
\mathrm{IT}_{\text {Standard }}=\mathrm{SDD}+\mathrm{SED}+\mathrm{X}
$$

where, $\mathrm{X}$ is a constant which represents the total propagation and processing delay from MME to HSS (100ms), MME to DNS (50ms) and PGW to PCRF (100ms) [12].

Thanks to the transition diagram for activation and deactivation procedures in [1], [4] and [13], SDD and SED can be derived, yielding:

$$
\begin{aligned}
& \mathrm{IT}_{\mathrm{Standard}}=2 *\left\{\mathrm{PD}_{(2 * \mathrm{UE}, 3 * \mathrm{eNB}, 2 * \mathrm{MME}, 2 * \mathrm{SGW}, \mathrm{LGG})}+\right. \\
& \left.\mathrm{LD}_{\left(3 * \mathrm{UE} \_\mathrm{NB}, 3 * \mathrm{eNB} \_\mathrm{MME}, 2 * \mathrm{MME} \_\mathrm{SGW}, 2 * \mathrm{SGW} \_ \text {LGW }\right)}\right\}+\mathrm{X}
\end{aligned}
$$

For the proposed scenario, the interruption time of SIPTO traffic is given by: 


$$
\begin{aligned}
& \mathrm{IT}_{\text {Proposed }}=\text { TED } \\
& =\mathrm{PD}_{(\mathrm{UE}, 2 * \mathrm{SeNB}, \mathrm{TeNB}, 3 * \mathrm{MME}, 2 * \text { TproxySGW,SLGW })}+ \\
& \text { LD }_{(2 * \text { MME_SeNB,SeNB_UE,4*MME_TeNB, }} \\
& \quad \text { UE_TeNB,2*TproxySGW_SLGW })
\end{aligned}
$$

We used values from 3GPP [14] for the processing delay on different nodes and the link delay between two different nodes. The propagation delay between different nodes is taken to be equal to $5 \mu \mathrm{sec} / \mathrm{km}$. In SIPTO@LN the LGW must be co-located with the base station (eNB). Based on this information we assume that the distance from eNB to the default SGW (co-located with PGW) and distance from SGW to MME equals $750 \mathrm{~km}$. We also assume that the link delay in proposed scenario from target proxy SGW to source LGW is $1.5 \mathrm{~ms}$. Therefore we obtain the results reported in Table I. The diagonal entries in Table I. represent the processing delay of nodes whilst the rest of the values correspond to the link delays.

TABLE I.
\begin{tabular}{|c|c|c|c|c|c|c|}
\hline & UE & eNB & MME & SGW & $\begin{array}{c}\text { Proxy } \\
\text { SGW }\end{array}$ & LGW \\
\hline UE & 3 & 2 & - & - & - & - \\
\hline eNB & 2 & 4 & 7.5 & 3.75 & 0 & 0 \\
\hline MME & - & 7.5 & 15 & 3.75 & 7.5 & - \\
\hline SGW & - & 3.75 & 3.75 & 10 & - & 3.75 \\
\hline $\begin{array}{c}\text { Proxy } \\
\text { SGW }\end{array}$ & - & 0 & 7.5 & - & 10 & 0 \\
\hline LGW & - & 0 & - & 3.75 & 0 & 10 \\
\hline
\end{tabular}

Given the numbers in Table I., applied to equations (2) and (3), we obtain the following results:

- the interruption time for standard scenario (IT Standard $_{\text {) }}$ approximately equals to $\mathbf{5 0 0} \mathbf{m s}$

- the interruption time for proposed scenario (IT Proposed $)$ is less than $150 \mathrm{~ms}$.

From these results, we can state that our proposed MPTCPbased SIPTO scenario enables operators to significantly reduce delay during the mobility of UEs having a SIPTO@LN connection. This allows seamless session continuity for users having ongoing video-streaming data taking into consideration that the delay budget for conversational video is estimated by $150 \mathrm{~ms}$ and non-conversational video is estimated by $300 \mathrm{~ms}$ [12]. Thus, obtained results using our method are fully compliant with the level of quality of service required for this kind of traffic.

The use of S1 based handover procedure for proposed architecture limits the possible infrastructure changes for signaling. Besides, the enhancement of the LGW with a proxy SGW function has allowed operators to use the LGW for real time sessions as well as for short-lived sessions at the same time. However, additional delay has been introduced to the network at the level of the (H)eNB for intercepting packets of both regular and offloaded sessions.

\section{CONCLUSION}

In the present paper, we have addressed the session continuity issue in SIPTO@LN reference model. We proposed an MPTCP based solution for mobility of users having ongoing sessions requiring IP address preservation. We enhanced the LGW with a Proxy SGW function. The MPTCP protocol is used to maintain the ongoing session even after the previous IP address becomes unreachable and UE acquires a new address. We have described how to maintain MPTCP signaling for creating new sub-flows and discarding old ones, relying on LTE architecture. This enhancement goes beyond the 3GPP study in [10] by using the LGW to offload longlived data sessions as well as short-lived data sessions. We have shown that our solution supports seamless mobility by significantly reducing the handover delay compared to the standard 3GPP SIPTO architecture. In future works we intend to evaluate the proposed solutions in an experimental setup in order to verify the feasibility of proposed solutions for real time scenario.

\section{REFERENCES}

[1] 3GPP, "General Packet Radio Service (GPRS) enhancements for Evolved Universal Terrestrial Radio Access Network (E-UTRAN) access," Technical specification, Release 13, TS 23.401, 2014.

[2] 3GPP, "Local IP Access and Selected IP Traffic Offload (LIPASIPTO)," Technical report, Release 10, TR 23.829, 2011.

[3] Eido, S., \& Gravey, A. (2014). How Much LTE Traffic Can Be Offloaded?. InAdvances in Communication Networking (pp. 48-58). Springer International Publishing.

[4] 3GPP, "Local IP Access (LIPA) mobility and Selected IP Traffic Overload (SIPTO) at the local network," Technical report, Release 12, TR 23.859, 2013.

[5] Korhonen, J., Soininen, J., Iisakkila, K., Bajko, G., Savolainen, T., \& Patil, B. (2012). RFC 6459: IPv6 in 3rd Generation Partnership Project (3GPP) Evolved Packet System (EPS).

[6] 3GPP, "Evolved Universal Terrestrial Radio Access (E-UTRA) and Evolved Universal Terrestrial Radio Access Network (E-UTRAN)," Technical report, Stage 2, Release 12, TS 36.300, 2014.

[7] Ford, A., Raiciu, C., Handley, M., \& Bonaventure, O. (2013). RFC 6824: TCP Extensions for Multipath Operation with Multiple Addresses.

[8] Mitharwal, P., Lohr, C., \& Gravey, A. (2014). Survey on Network Interface Selection in Multihomed Mobile Networks. In Advances in Communication Networking (pp. 134-146).

[9] Paasch, C., Detal, G., Duchene, F., Raiciu, C., \& Bonaventure, O. (2012, August). Exploring mobile/WiFi handover with multipath TCP. In Proceedings of the 2012 ACM SIGCOMM workshop on Cellular networks: operations, challenges, and future design (pp. 31-36). ACM.

[10] 3GPP, "Study on co-ordinated Packet data network GateWay (PGW) Change for Selected IP Traffic Offload (CSIPTO)," Technical report, Release 13, TR 22.828, 2014.

[11] Nguyen, Tien-Thinh, and Christian Bonnet. "A Hybrid CentralizedDistributed Mobility Management for Supporting Highly Mobile Users."

[12] Savic, Z. "LTE Design and Deployement Strategies," Cisco, 2011.

[13] Gupta, R., and Rastogi, N., "LTE Advanced - LIPA and SIPTO," White Paper, Aricent 2012.

[14] 3GPP, "Feasibility study for evolved Universal Terrestrial Radio Access (UTRA) and Universal Terrestrial Radio Access Network (UTRAN)," Technical report, Release 12, TR 25.912, 2014. 